\title{
A neural network simulator for supercomputers
}

\author{
WILLIAM S. MAKI and ADEL M. ABUNAWASS \\ North Dakota State University, Fargo, North Dakota
}

\begin{abstract}
Connectionist modeling is computationally intensive. Until parallel computers become more widely available, supercomputing resources can be exploited. This paper describes a neural network simulator (NNS) written in FORTRAN for supercomputers. The present simulation engine consists of code for the backpropagation method of changing weights in connectionist models. A file interface reports simulation results in a variety of formats. The file interface also contains an interpreter for an input file through which the network structure is defined, the problem is represented, and various parameters are set. The input-file syntax is described in detail. NNS has been used both as an instructional aid and as a research tool. A simulation of "recovery of unrehearsed associations" is used to illustrate the use of the input file and to demonstrate the performance of NNS. Versions of NNS have been written for the Cray X-MP/48 and for the IBM 3090.
\end{abstract}

In his presidential address to the Society for Computers in Psychology, Walter Schneider explored the profound and far-reaching impact of connectionist models on psychology (Schneider, 1987). The interest in connectionist models, if anything, has intensified; a recent issue of the Journal of Memory and Language was devoted to connectionist models, and the stream of books, journal articles, and papers and symposia at the meetings of the Psychonomic and Cognitive Science societies continues unabated. A collection of reprints covering important developments in the history of connectionism has been assembled by Anderson and Rosenfeld (1988), and critical assessments of the strengths and weaknesses of connectionist approaches to cognition have appeared (Holyoak, 1987; McClelland, 1988; Minsky \& Papert, 1988; Pinker \& Mehler, 1988; Waltz \& Feldman, 1988).

Connectionism, also referred to by the terms neural networks and parallel distributed processing (PDP), was introduced to the readers of Psychological Review 30 years ago by Frank Rosenblatt in the form of the "perceptron" (Rosenblatt, 1958). Despite the promise of brain-style models of perception and learning, interest in perceptrons waned during the ensuing years for a host of arguable reasons, including limitations on what perceptrons could compute (Minsky \& Papert, 1988) and lack of computa-

NNS was developed on the Cray X-MP/48 under the auspices of the National Center for Supercomputing Applications Academic Affiliates Program. The simulations reported in this paper were performed on the Cray X-MP/48 and on the IBM 3090-120E at North Dakota State University; the vector processor was provided by IBM through a joint study agreement with North Dakota State University. NNS was originally conceived by William Maki. The present version of NNS was designed and coded by Adel Abunawass. A. M. Abunawass is in the Department of Computer Science at North Dakota State. Requests for reprints should be sent to William S. Maki, Department of Psychology, North Dakota State University, Fargo, ND 58105-5075. tional resources needed to simulate the parallel processing inherent in neural-type networks (Rosenberg \& Blelloch, 1988). By the early 1980 s, however, interest among psychologists in connectionist modeling had been rekindled.

The perceptron, a computational model, captured some of the general features of what was then known about neurophysiology. In its simplest form, several neural-like (S) units were connected to another $(\mathrm{R})$ unit by modifiable connections. In more complex networks, $S$ units were connected to intervening A units, which in turn connected to $R$ units (Rosenblatt, 1962). A very small network is depicted in Figure 1. Translating to modern terms, the network consists of two input (S) units, four hidden (A) units, and one output ( $R$ ) unit.

The modifiability of the connections (weights) between units is an important feature of such networks. Widrow and Hoff (1960) devised a powerful learning rule, according to which the difference between the observed value of an output unit and its desired value (error) is used to modify the strengths of the connections feeding the output unit. The rule is similar to the well-known model of classical conditioning developed some years later by Rescorla and Wagner (1972; see Sutton \& Barto, 1981). A more recent version, the generalized delta rule, employing backpropagation of errors, was derived by Rumelhart, Hinton, and Williams (1986a, 1986b). Backpropagation, like other hill-climbing methods, has its problems (Holyoak, 1987; McCloskey \& Cohen, 1987; Minsky \& Papert, 1988; Sutton, 1986), and it represents just one class of models (cf. Hinton \& Sejnowski, 1986). However, backpropagation remains a simple and popular computational basis for simulating neural networks. The relationship of the generalized delta rule to previous models of associative learning (see Gluck \& Bower, 1988) motivated work on the program described in this paper. 


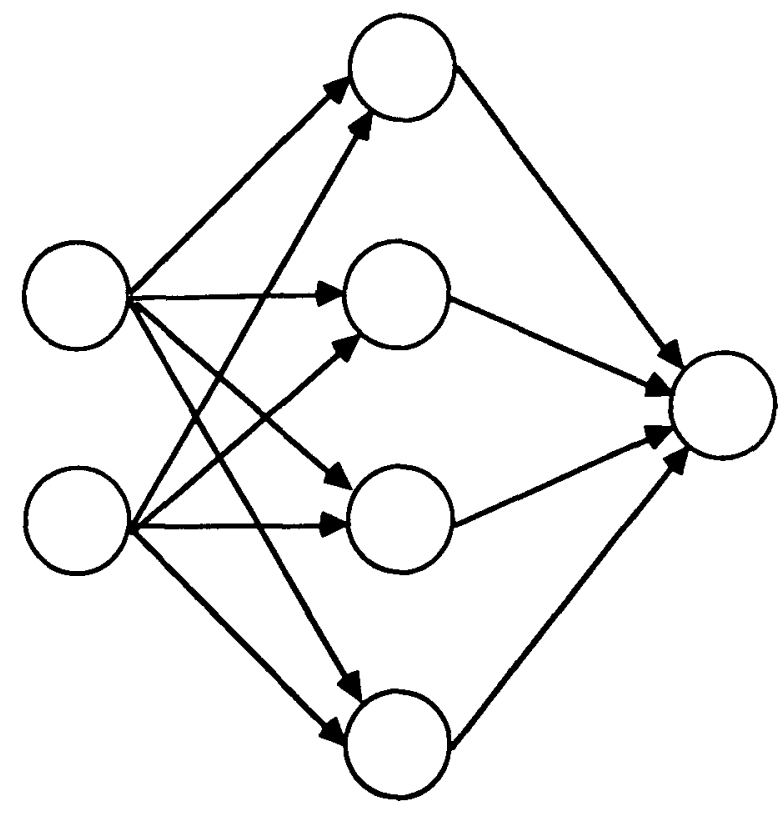

Figure 1. An example of a connectionist network that solves the XOR problem. The circles represent neural-like units and the arrows represent connections between units. The network is composed of (from left to right) two input units, four hidden units, and one output unit.

\section{Computational Resources for Connectionist Modeling}

When the backpropagation technique is used, the small network sketched in Figure 1 can learn an interesting problem called the exclusive or (XOR; see Rumelhart et al., 1986a). The XOR is nonlinear and consists of four input/output patterns. A zero applied to each input is associated with an expected output of zero $(00 \rightarrow 0)$; the other three patterns are $01 \rightarrow 1,10 \rightarrow 1$, and $11 \rightarrow 0$. However, most problems require much larger networks. When the XOR function is expanded to describe the structure of the conditional discrimination problem learned by animals known as matching-to-sample (Maki, 1988), more inputs and outputs are needed, with a correspondingly larger number of connections. Simulations of paired-associate learning represent pairs of unrelated words as pairs of random vectors several bits in length, and the coding capacity of the network is enhanced by increasing the number of hidden units; the resulting network contains thousands of connections (e.g., see Hinton \& Plaut, 1987; McCloskey \& Cohen, 1987). The NETtalk program, which learns to translate written words into their phonetic equivalents, contains over 13,000 connections (Rosenberg \& Blelloch, 1988). Clearly, as connectionist models are scaled up to simulate "real" phenomena, the computational demands rise dramatically.

Several computing approaches to neural networks are now available. Some are suitable for small demonstrations; others can handle large research-grade simulations. Halff (1987) used a commercially available spreadsheet on a personal computer to simulate low-level visual processes. McClelland and Rumelhart (1988b) developed software to accompany their PDP volumes; the software, written in $\mathrm{C}$, runs on personal (micro) computers and larger computers like the digital Equipment Corporation VAX and is sold with a manual of "explorations" (McClelland \& Rumelhart, 1988a). Zipser and Rabin (1986) described P3, a neural network simulation language written in LISP and implemented on a LISP machine. Neurocomputers are hardware solutions to implementing neural networks and are presently designed as coprocessors hosted by, for example, personal computers running DOS; these systems cost several thousands of dollars (see Hecht-Nielsen, 1988, for a review).

The ideal machine for simulating brain-like, massively parallel processes would appear to be a large parallel computer (see Rosenberg \& Blelloch, 1988). However, such machines are not widely available. An alternative approach would be to use a supercomputer that provides some of the needed storage size and speed. The need for supercomputing resources to support connectionist simulations was anticipated by Green, Null, Furnas, Hagen, and Rumelhart (1985). Access to supercomputers (as of this writing) is available through the nation's supercomputing centers funded by the National Science Foundation. The principle language used on supercomputers is FORTRAN.

\section{The Neural Network Simulator for Supercomputers (NNS)}

The program reported here was written in FORTRAN and was meant for use on large computers such as the Cray X-MP/48 or IBM 3090 series. We have tried to design NNS for portability between product lines and compatibility within product lines. The simulator consists of two main parts: a collection of routines known as the simulation engine, and another collection of routines known as the file interface.

The simulation engine contains several arrays in which information about the network is stored; learning-rate parameters, current activation values for each unit, current weights and weight changes, and matrices containing the input and output patterns are among the many types of data kept in memory. The actual engine consists of several FORTRAN subroutines. Some routines deal with initialization tasks like random-number generation. Others accomplish the forward (input to output) spread of activation through the network. Another group of routines computes errors and accomplishes backpropagation. Every effort has been made to make the code as modular as possible; the result is that modifications and/or extensions to the engine are easily installed. Although we have not yet done so, in principle it should be possible to add an entirely different type of learning algorithm (like the Boltzmann machine; Hinton \& Sejnowski, 1986) while making use of existing data structures and the file interface.

The file interface contains two groups of subroutines. One set of routines handles the chores associated with 
producing output files containing the results of the simulation. The formats of the output will be discussed in the context of the input file. The input file is the means by which the user of NNS communicates the structure of the network, describes the nature of the problem to be solved, and sets the parameters required by the backpropagation algorithm as well as those peculiar to NNS. The input file is described below in some detail so as to suggest more concretely some of the capabilties and limitations of NNS.

\section{The NNS Input File}

Each NNS run is controlled by an input file. A typical file consists of many keywords, each followed by one or more lines of specifications. The structure of this file and the functions of its various parts will be illustrated with the network shown in Figure 1. The problem to be learned is the XOR. (The general form of NNS input file syntax is displayed in the Appendix.) In what follows here, we will introduce the keywords by listing the text of the input file followed by qualifying remarks. Occasionally, additional comments preceded by semicolons will be made on the right side of the listing.

Network definition. Defining the network begins with a sketch similar to that in Figure 1. The units are then assigned identifying numbers. In NNS, units are numbered sequentially beginning with the input units. The two input units in Figure 1 are thus assigned identities of 1 and 2 . The four hidden units are numbers $3,4,5$, and 6 . The output unit is number 7 .

We then begin the input file by informing NNS of the number of units in our network,

\section{UNITS}

\section{7}

we declare the number of input units,

\section{INPUT_UNITS}

\section{2}

and we identify the output units by the OUTPUT_UNITS keyword followed by the number of the first output unit and the number of the last output unit:

\section{OUTPUT_UNITS}

7,7

(in this case, there is only one output unit so the numbers of the starting and last output units are the same).

The structure of the network is described using the CONNECTIONS keyword in either of two ways. The first method of describing a network is the most flexible but also the most cumbersome. One can simply declare each connection in the network. The first line following the keyword contains the number of connections, and each connection is then declared, one connection per line. The form of each declaration is unit(i), unit(j), starting weight, initial weight change, and a flag. If the flag is zero, the weight is modifiable; if the flag is set (to one), the weight is fixed.

\section{CONNECTIONS}

12

$1,3,0,0,0$

$1,4,0,0,0$

$1,5,0,0,0$

$1,6,0,0,0$

$2,3,0,0,0$

$2,4,0,0,0$

$2,5,0,0,0$

$2,6,0,0,0$

$3,7,0,0,0$

$4,7,0,0,0$

$5,7,0,0,0$

$6,7,0,0,0$

Note that this method can be used to specify a network with incomplete connectivity, and also to specify (hardwired) connections with unchanging weights.

The second method is meant to be used when successive clusters of units are fully interconnected. On the first line following the keyword, the minus sign indicates that each layer of connections is complete, and the integer is the number of rows (layers) to follow. The next line specifies all possible connections between the input units (from Unit 1 to Unit 2) and the hidden units (from 3 to 6). The third line does the same for the hidden units (from 3 to 6 ) and the output unit (from 7 to 7).

\section{CONNECTIONS}

$-2$

$1,2,3,6$

$3,6,7,7$

This method of specifying connections is shorter than the method first described, but it can only be used when full connectivity throughout the network is desired.

The THRESHOLDS keyword can be used to declare unit biases (theta values; see Rumelhart et al., 1986a). This optional keyword works like the long method of declaring connections, and its syntax is described in the Appendix.

The network must be further articulated by declaring the structure of the hidden units.

\section{HIDDEN_L_LAYERS \\ 1 \\ 3,6 \\ ;number of hidden layers ;inclusive unit numbers}

Here there is only a single hidden layer. If a network contained more than one such layer, the number of hidden layers would be larger, and additional rows containing unit numbers would follow.

In the final step of network description, the simulation engine is informed of the types of calculations to be performed on the various connections. The most common type of connection is found where units in layer $\mathbf{N}$ feed forward to units in layer $N+1$; we refer to the computation involved in such connections as Calculation Type 3 . The sample network considered here (Figure 1) contains 
feed-forward connections only, so the specification is simple.

\section{DRIVER \\ 2 ;two lines follow \\ 0,3,8 ;Layer 0, Calculation Type 3, 8 connections \\ 1,3,4 ;Layer 1, Calculation Type 3, 4 connections}

In recurrent networks (see Rumelhart et al., 1986a), units may be reciprocally connected (NNS Calculation Type 1) and/or self-connected (Calculation Type 2). The calculation types specify the order in which calculations are performed and so should appear, within each layer, in numerical order. Numbering of layers begins with 0 (which refers to the connections emanating from input units).

Simulation identification and definition. An optional keyword can be used to generate brief (20-character) names for simulations.

\section{SIMULATION_NAME XOR_EXAMPLE}

Provisions have been made to expand NNS to include variations on the backpropagation theme like the sequential network (Jordan, 1986). Two keywords allow for control over the subroutines selected for use by the simulation engine. The SIMULATION_TYPE keyword was intended to allow for different methods of computing error. At present, SIMULATION__TYPE should be set to 1 , indicating that the error for each input/output pattern is determined by the difference between expected activations (teaching inputs) and observed activations of output units.

\section{SIMULATION_TYPE}

\section{1}

However, processing can be varied now by the INPUT TYPE keyword. When INPUT_TYPE is 1 , weights are changed after each input/output pattern and patterns are presented in randomized blocks (just as we might suppose learning to occur following individual experimental trials). When INPUT_TYPE is 3 , weights are changed after each sweep through the entire set of patterns (as is common in simulation work of this type; see, e.g., Hinton \& Plaut, 1987).

\section{INPUT_TYPE \\ 1}

The actual structure of the problem to be simulated is described by the INPUT_PATTERNS keyword. Below is an example of the description of the XOR problem containing the input/output patterns 00-0, 01-1, 10-1, and 11-1. Note that an additional digit (1) is appended to each input pattern; the additional digit anticipates future enhancements of NNS (e.g., allowing each input/output pattern to be repeated a specified number of times).

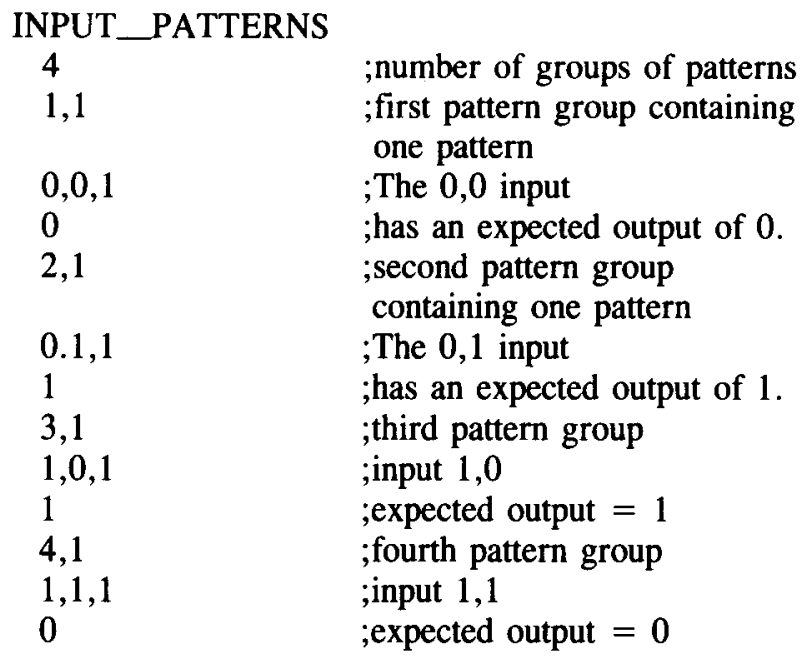

Because we wish to run a single simulation in this example, we declare the number of simulations as 1 .

\section{SIMULATIONS \\ 1}

The number of simulations can be set to any desired number of replications.

The simulation of transfer of training (as discussed later) is complicated by multiple stages of the experimental design of a transfer experiment. In a simulation of transfer, the initial portion of the input file must be interpreted and the first simulation executed before interpreting the rest of the file. The optional RUNSIM keyword instructs NNS to perform the simulation before continuing with interpretation of the input file (when used, RUNSIM appears on a single line).

Report generation. The output of NNS takes the form of (optional) files. Several keywords allow the user to customize the output of NNS. The syntax of all the keywords is contained in the Appendix. For the present illustration, we focus on those keywords which provide for testing the network's ability to generalize by presenting input patterns not used during training.

The syntax of the GENERALIZATION keyword is identical to that of the INPUT_PATTERNS keyword. To test the network's reaction during and/or after training to the full complement of training patterns, we include the following.

$\begin{array}{ll}\text { GENERALIZATION } & \\ 4 & \text {;number of groups of patterns } \\ 1,1 & \text {;first group with one pattern } \\ 0,0,1 & \text {;input }=0,0 \\ 0 & \text {;expected output }=0 \\ 2,1 & \text {;etc. } \\ 0,1,1 & \\ 1 & \\ 3,1 & \\ 1,0,1 & \\ 1 & \\ 4,1 & \end{array}$




$$
1,1,1
$$

0

Note that the expected output is required here but in fact only serves to remind the user of how the network should be responding to the various input patterns. Note also that the choice of input/output patterns for inclusion in the GENERALIZATION paragraph is arbitrary; any pattern (used in training or not) can be included.

The type of report generated is controlled by the GENERALIZATION_REPORT keyword. If the type is set to 1 , a single line in comma-delimited format is produced for each output unit in each pattern, which contains the simulation name, the group number, the pattern number, the output unit number, and the observed and expected activation values. If the type is set to 2 , a much more extensive report is written to a different file, which contains the values of all the weights and unit biases as well as the information generated in the Type 1 file. If the type is set to 3 , both fields are written. Types 4 and 5 produce abbreviated reports designed for downloading to commercially available spreadsheets (e.g., Microsoft Excel) and differ only in delimiters (comma vs. tab). In this example, one of the latter types of report (Type 5) is desired.

\section{GENERALIZATION_REPORT 5}

The frequency with which the reports are appended to their respective files is governed by the REPORTING keyword. In the example below, we wish to have a report generated every 100 sweeps through the training set.

\section{REPORTING}

100

By default, a report is generated when training is completed, so the REPORTING keyword is optional.

The reporting from NNS is an area of ongoing development. Connectionist simulations can generate huge amounts of data that need to be creatively packaged in ways that can easily be consumed by scientists (e.g., Zipser \& Rabin, 1986). To this end we are designing output files that periodically report values of selected units and weights in order to depict NNS output graphically. Some of the capabilities in this regard are suggested by the PROBE_UNITS and PROBE_WEIGHTS keywords described in the Appendix.

The PDP_ _ REPORT keyword adds an interesting capability to NNS. If the flag for this keyword is set to 1 , then a file is generated in the format used by McClelland and Rumelhart (1988a) in their weights (*.wts) files. This means that training of a network could be accomplished very rapidly on the supercomputer and that the behavior of the network could then be interactively studied on a personal computer or a workstation using the McClellandRumelhart explorations package.
Random numbers. It is customary to begin a simulation with starting weights set to relatively small random numbers. The RANDOM, MEAN, and LIMIT keywords specify the type of distribution $(0=$ normal, $1=$ uniform), and two parameters - the mean and the limit. With a normal distribution, the limit is in fact the value of the standard deviation; a uniform distribution is bounded by mean \pm limit. In this example, we specify a uniform distribution with a limit of 0.3 .

\section{RANDOM \\ 1 \\ MEAN \\ 0.0 \\ LIMIT \\ 0.3}

The seed for the random number generator in use by the host system can be controlled by the SEED keyword. If the specified seed is 0.0 , then a seed based on the present date and time (system variables) is computed. In any event, the seed is saved in an output file, so that the starting state of the network can be restored at some later time.

\section{SEED}

$123457.0 \quad$;a predefined seed is used here.

Some useful options. The behavior of the networks in simulations of transfer of training and retroactive inhibition (McCloskey \& Cohen, 1987) is an important area of study and one of current active interest in our laboratory. Such studies can be accomplished with NNS by means of using the KEEP_WEIGHTS keyword (in conjunction with the RUNSIM keyword already described). An abbreviated example follows.

$\begin{array}{ll}\ldots & \begin{array}{l}\text {;keywords describing } \\ \text { original training. } \\ \text {;instruction to run } \\ \text { simulation. } \\ \text { RUNSIM }\end{array} \\ \text { KEEP_WEIGHTS } & \begin{array}{l}\text {;N told to use existing } \\ \text { weights for remainder } \\ \text { of simulation. } \\ \text {;more keywords } \\ \text { describing transfer test }\end{array}\end{array}$

The KEEP _ WEIGHTS keyword, with a flag of 1, causes NNS to retain preexisting weights during subsequent phases of the simulation. When the flag is set to 0 , the weights are reset to values specified by the RANDOM, MEAN, and LIMIT keywords.

The resilience of connectionist networks has been demonstrated by simulated neural damage (e.g., Anderson, 1983; Hinton \& Sejnowski, 1986). NNS includes the tools necessary to perform such studies. The MODIFY_UNITS is used to create "lesions" by permanently disabling a set of units; the specified units then produce zero outputs. The pattern of weights in the network can be distorted by using the NOISE keyword; 
random values specified in the parameters of the keyword are added to each weight in the network. Also, one can preserve already specified connections by the KEEP_WEIGHTS keyword and then selectively alter connections by the long form of the CONNECTIONS keyword.

Learning parameters. The generalized delta rule (Rumelhart et al., 1986a, 1986b) contains two rate parameters. For example, to change the weight between an output unit $j$ and some other unit $i$, we compute

$$
\Delta w_{i, j, t}=\eta o_{i} \delta_{j}
$$

and

$$
w_{i, j}=w_{i, j, t-1}+\Delta w_{i, j, t}+\alpha \Delta w_{i, j, t-1} .
$$

Some values are computed during the course of the simulation: $w_{i, j}$ represents the weight on the connections between units $i$ and $j$ at time $t$ (or $t-1$ ), $o_{i}$ is the activation of unit $i$, and $\delta_{j}$ is the error associated with unit $j$. The learning rate parameter $\eta$ and the momentum parameter $\alpha$ are set in the input file as follows:

ETA

$$
0.25
$$

ALPHA

0.90

Stopping criteria. Three events can be detected and used to stop an NNS simulation. First, a maximum number of sweeps may be reached. This maximum is set by the MAXNUM keyword. In this example, NNS will halt after 5,000 sweeps:

\section{MAXNUM 5000}

NNS can also stop if the absolute error (the absolute value of the difference between the desired and obtained activation, $\left.\left|t_{j}-o_{j}\right|\right)$ for each output unit is less than a value set by the ERROR keyword when the ERROR_CRIT keyword is set to 1 . This is the method selected in the present example. Here the activation of each and every output unit in the entire network must be within 0.1 of its expected value (teaching input) to cause the simulation to terminate:

\section{ERROR_CRIT \\ 1 \\ ERROR}

0.1

NNS can stop in a third way: if the ERROR CRIT parameter is set to 2 , the simulation will terminate if the mean value of $\left|t_{j}-o_{j}\right|$, averaged over all output units, is less than the value specified by the ERROR keyword.

Finally, it should be noted that saying a simulation "terminates" means only that NNS writes whatever final reports have been specified and then searches for additional keywords in the input file. An end-of-file condition encountered during interpretation forces a RUNSIM and only then does NNS truly halt.

\section{"Recovery of Unrehearsed Associations": A Simulation Using NNS}

A very interesting finding has emerged from recent work on connectionist models. Neural networks turn out to be very susceptible to retroactive interference from learning new associations (McCloskey \& Cohen, 1987). Of more interest here is the report that the retraining of just a few of the originally learned associations promotes substantial recovery of nonretrained associations. Hinton and Plaut (1987) constructed a network of 10 input units, 100 hidden units, and 10 output units. Each of the 2,000 connections was composed of both fast and slow weights. The network was taught a list of associations between the random 10-bit vectors contained in 100 pairs (original learning). Then the same network was presented with a list of five new vector pairs (interpolated learning). $\mathrm{Fi}$ nally, a fraction ( $10 \%$ or $50 \%$ ) of the pairs on the original list were again presented for 20 retraining trials. At the beginning of retraining, the network showed the deleterious effects of the interference in a relatively large discrepancy between the observed activation levels of output units and those expected on the basis of original learning. As retraining progressed, the error was reduced for retrained pairs, but it was also reduced for nonretrained pairs.

We have performed several simulations as preliminary steps to a fuller analysis of the recovery phenomenon reported by Hinton and Plaut (1987). (In the present context, the simulations provide an illustration of NNS in an ongoing research program.) The simulations differed from that of Hinton and Plaut (1987) in that each connection had a single (slow) weight. Otherwise, the details of the initial simulation were crafted to be as identical as possible to those reported by Hinton and Plaut.

This simulation, performed on the Cray X-MP/48, took $305 \mathrm{sec}$; the same simulation performed on the IBM 3090$120 \mathrm{E}$ took $29.5 \mathrm{~min}$. One can also express performance in terms of the number of connections computed per unit time. In this simulation, the network contained 2,000 connections. In original learning, those connections were computed for each of 100 patterns over 1,300 sweeps $(2,000 \times 100 \times 1,300)$. The corresponding calculations for interpolated learning and retraining are 2,000 $\times 5 \times$ 400 and $2,000 \times 50 \times 20$. This yields a total of $266 \mathrm{mil}-$ lion connections computed during the course of the simulation. Dividing by the computing times, we get 872,131 connections/sec for the Cray and 150,282 connections/sec for the IBM. In fact, these are conservative estimates of performance, because we did not measure connection computation time independently of the computation associated with interpreting the input file and report writing. Moreover, these connections were all with learning (forward activation plus backpropagation), and an additional 4.5 million forward connections were made in the course of generalization reporting (but not included in the performance analysis). Even so, these performances are on the high end of those of the machines summarized by Rosenberg and Blelloch (1988) and compare favorably 


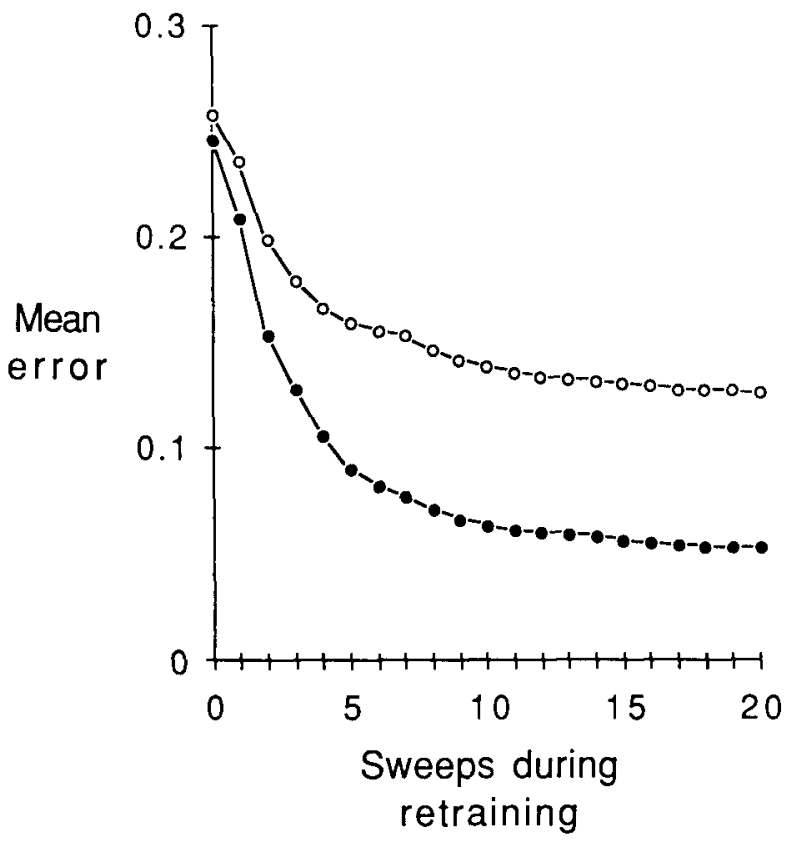

Figure 2. Mean absolute error for eight retrained and eight nonretrained pairs during 20 retraining trials (sweeps) following interpolated learning. Data are averages from 10 simulations performed with NNS, using input files similar to that shown in Table 1. Solid symbols represent data from retrained pairs; open symbols represent data from nonretrained pairs.

with the performance of a $16 \mathrm{~K}$-processor parallel computer. Obviously, performance would be even better with a faster machine like the Cray 2 or with additional vector processors added to the IBM 3090.

In another set of simulations we obtained the recovery effect but in much less computing time (about $37 \mathrm{sec}$ per simulation). The network was scaled down to 8 input units, 64 hidden units, and 8 output units. In each of 10 simulations, the network was first trained to associate 16 pairs of 8-bit random vectors (300 sweeps through the entire list). Then the network was trained on a list of four new vectors ( 100 sweeps) and then retrained on eight pairs from the original list ( 20 sweeps). Throughout, $\eta=0.05$ and $\alpha=0.90$. The 10 simulations differed only with respect to list and random number seeds; each of five lists was used twice. Other details of the simulations can be found in the sample input file displayed in Table 1.

By the end of original learning, the network had learned the associations; the average error of an output unit, $\left|t_{j}-o_{j}\right|$, was less than 0.05 . The interpolated training on the four new pairs caused retroactive interference as gauged by the increased error $(0.25)$. The average error associated with the eight pairs that were present during retraining is shown in Figure 2; the figure also displays the average error associated with those pairs that were not retrained. Retraining restored accurate performance on the retrained pairs. Retraining also improved performance on the nonretrained pairs; the recovery was not complete, but the effect was noted in each one of the 10 simulations.

\section{Experience with NNS and Usability Issues}

We now have over a year of experience working with NNS. NNS has been used on both the Cray X-MP/48 run interactively under CTSS at the National Center for Supercomputing Applications and on the IBM 3090-120E run in batch mode under MVS/XA at North Dakota State University. The two versions differ only in two respects. First, the details of file input/output depends on the operating system, so some recoding needs to be done prior to installing NNS on machines with operating systems other than those just mentioned. Second, generation of random numbers and access to system date and time variables depends on the version of FORTRAN and the available mathematical subroutine libraries (which vary from system to system); these routines also need to be examined

Table 1

NNS Input-File Specifications for "Recovery" Simulation

CONNECTIONS

$-2$

$1,8,9,72$

$9,72,73,80$

UNITS

80

INPUT_UNITS

8

OUTPUT_UNITS

73,80

HIDDEN_LAYERS

1

9,72
; Short form of CONNECTIONS

TONNECTIONS




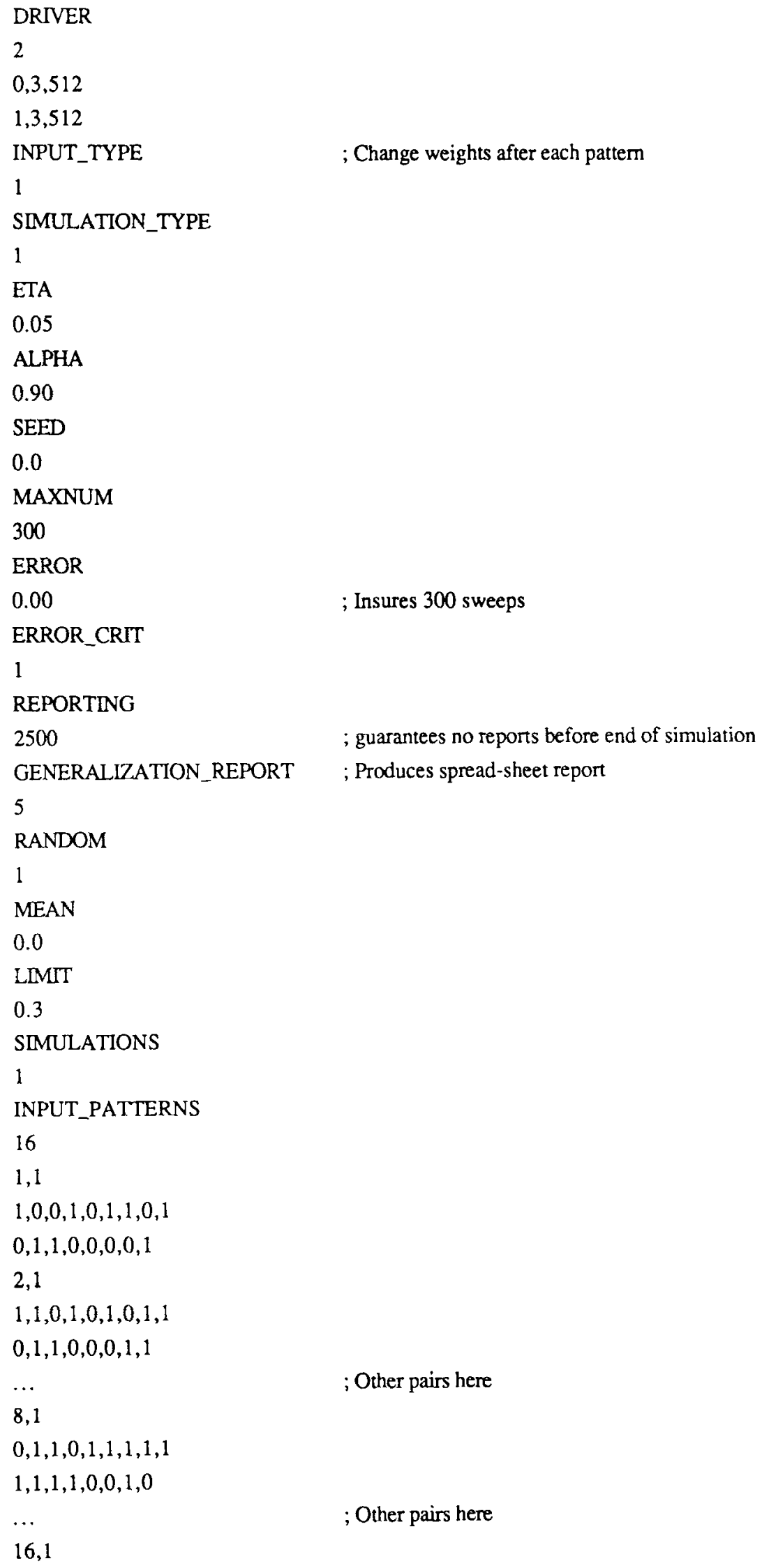




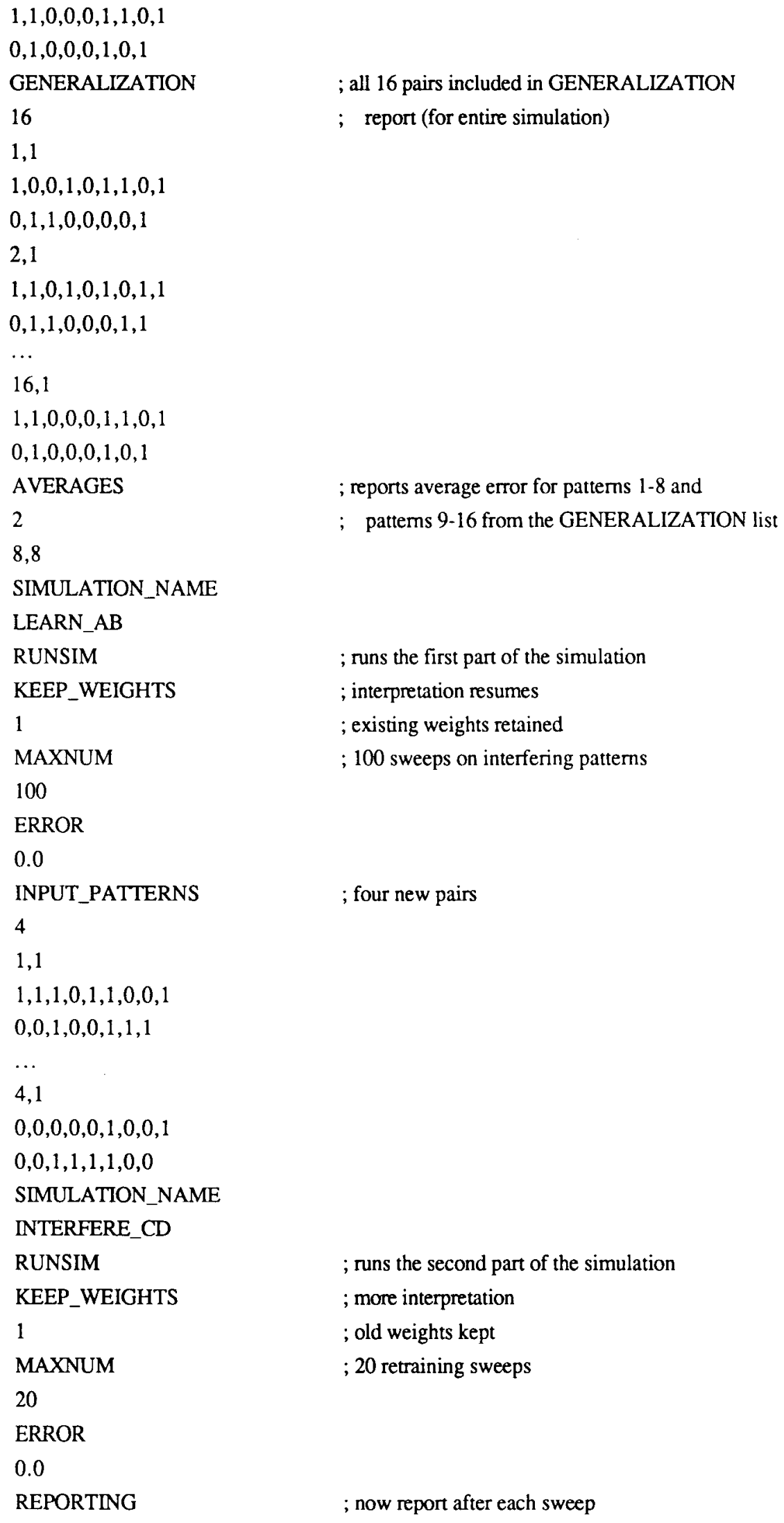




\section{1}

SIMULATIONS

1

INPUT_PATTERNS

8

1,1

$1,0,0,1,0,1,1,0,1$

$0,1,1,0,0,0,0,1$

2,1

$1,1,0,1,0,1,0,1,1$

$0,1,1,0,0,0,1,1$

$\ldots$

8,1

$0,1,1,0,1,1,1,1,1$

$1,1,1,1,0,0,1,0$

SIMULATION_NAME

RETRAIN_HALF_AB

; end of file -- run last part of simulation

and perhaps recoded prior to installation of NNS. We guess that a programmer with some experience in FORTRAN could have NNS installed within an hour or two.

We are continually using NNS as a research tool for simulations of the sort described in this paper, and the first author used an earlier version of NNS as an instructional aid in a course on neural networks. Many features of the input-file keyword specifications resulted from this experience. For example, the short form of the CONNECTIONS keyword was devised because of the need to concisely specify large networks. We found that providing some assistance with input-file construction is important, particularly so to first-time users of NNS.

\section{Availability}

The source code for NNS can be obtained by electronic mail (BITNET) from the first author: send electronic requests to nu021116@ndsuvml; specify the computer and operating system on which NNS will be installed. Current NNS documentation can be obtained by sending a formatted diskette (either 3.5- or 5.25-in.) and a selfaddressed, stamped mailer to the first author.

\section{REFERENCES}

ANDERSON, J. A. (1983). Cognitive and psychological computation with neural models. IEEE Transactions on Systems, Man, \& Cybernetics, 13, 799-815.

ANDERSON, J. A., \& RoSENFELd, E. (Eds.) (1988). Neurocomputing: Foundations of research. Cambridge, MA: MIT Press.

GlucK, M. A., \& BowER, G. H. (1988). Evaluating an adaptive network model of human learning. Journal of Memory \& Language, 27, 166-195.
Green, B. F., Null, C. H., Furnas, G., Hagen, M., \& RumelHART, D. (1985). Advanced computing in psychology. Behavior Research Methods, Instruments, \& Computers, 17, 331-338.

HaLfF, L. A. (1987). A spreadsheet simulation of the early stages of visual information processing. Behavior Research Methods, Instruments, \& Computers, 19, 117-122.

HeCht-NiElsen, R. (1988, March). Neurocomputing: Picking the human brain. IEEE Spectrum, pp. 36-41.

Hinton, G., \& SEJNOwski, T. J. (1986). Learning and relearning in Boltzmann machines. In D. E. Rumelhart, J. L. McClelland, \& the PDP Research Group (Eds.), Parallel distributed processing: Explorations in the microstructure of cognition: Vol. 1. Foundations (pp. 282-317). Cambridge, MA: MIT Press.

Hinton, G. E., \& Plaut, D. C. (1987). Using fast weights to deblur old memories. Proceedings of the Ninth Annual Conference of the Cognitive Science Society (pp. 177-186). Hillsdale, NJ: Erlbaum.

HolyoAK, K. (1987). A connectionist view of cognition. Science, 236, 992-996.

JoRdAN, M. I. (1986). Attractor dynamics and parallelism in a connectionist sequential machine. Proceedings of the Eighth Annual Conference of the Cognitive Science Society (pp. 531-546). Hillsdale, NJ: Erlbaum.

MakI, W. S. (1988, November). Extending connectionist models to animal cognition. Paper presented at the meeting of the Psychonomic Society, Chicago, IL.

McClelland, J. L. (1988). Connectionist models and psychological evidence. Journal of Memory \& Language, 27, 107-123.

McClelland, J. L., \& RumelhaRT, D. E. (1988a). Explorations in parallel distributed processing: $A$ handbook of models, programs, and exercises. Cambridge, MA: MIT Press.

McClelland, J. L., \&umelhart, D. E. (1988b). A simulation-based tutorial system for exploring parallel distributed processing. Behavior Research Methods, Instruments, \& Computers, 20, 263-275.

MCCloskey, M., \& Cohen, N. J. (1987, November). The sequential learning problem in connectionist modeling. Paper presented at the meeting of the Psychonomic Society, Seattle, WA.

Minsky, M. L., \& PAPERT, S. A (1988). Perceptrons: An introduction to computational geometry (expanded edition). Cambridge, MA: MIT Press. 
Pinker, S., \& Mehler, J. (1988). Connections and symbols. Cambridge, MA: MIT Press.

Rescorla, R. A., \& Wagner, A. R. (1972). A theory of Pavlovian conditioning: Variations in the effectiveness of reinforcement and nonreinforcement. In A. H. Black \& W. F. Prokasy (Eds.), Classical conditioning II: Current theory and research (pp. 64-99). New York: Appleton-Century-Crofts.

RosenberG, C. R., \& BLELLOCH, G. (1988). An implementation of network learning on the connection machine. In D. Waltz \& J. A. Feldman (Eds.), Connectionist models and their implications: Readings from cognitive science (pp. 329-340). Norwood, NJ: Ablex.

Rosenblatt, F. (1958). The perceptron: A probabilistic model for in formation storage and organization in the brain. Psychological Review, 65, 386-408

Rosenblatt, F. (1962). Principles of neurodynamics: Perceptrons and the theory of brain mechanisms. Washington, DC: Spartan.

RumelharT, D. E., Hinton, G. E., \& Wiluams, R. J. (1986a). Leaming internal representations by error propagation. In D. E. Rumelhart, J. L. McClelland, \& the PDP Research Group (Eds.), Parallel distributed processing: Explorations in the microstructure of cognition: Vol. 1. Foundations (pp. 318-362). Cambridge, MA: MIT Press.

Rumelhart, D. E., Hinton, G. E., \& Williams, R. J. (1986b). Leam- ing representations by back-propagating errors. Nature, 323,533 536.

SCHNEIDER, W. (1987). Connectionism: Is it a paradigm shift for psychology? Behavior Research Methods, Instruments, \& Computers, 19. 73-83.

SutTon, R. S. (1986). Two problems with backpropagation and other steepest-descent learning procedures for networks. Proceedings of the Eighth Annual Conference of the Cognitive Science Society (pp. 823831). Hillsdale, NJ: Erlbaum.

SutTon, R. S., \& Barto, A. G. (1981). Toward a modern theory of adaptive networks: Expectation and prediction. Psychological Review, 88, $135-170$.

Waltz, D., \& Feldman, J. (1988). Connectionist models and their implications. In D. Waltz \& J. A. Feldman (Eds.), Connectionist models and their implications: Readings from cognitive science (pp. 111). Norwood, NJ: Ablex.

Widrow, B. E., \& HoFf, M. E. (1960). Adaptive switching circuits. IRE WESCON Convention Record, 4, 96-104.

ZIPSER, D., \& RABIN, D. (1986). P3: A parallel network simulating system. In D. E. Rumelhart, J. L. McClelland, \& the PDP Research Group (Eds.), Parallel distributed processing: Explorations in the microstructure of cognition: Vol. I. Foundations (pp. 488-506). Cambridge, MA: MIT Press.

\section{APPENDIX \\ Neural Network Simulator Keyword Syntax}

A. Network Definition

1. Total Number of Units

UNITS

total_number_of_units

2. Total Number of Input Units

INPUT_UNITS

number_of_input_units

3. Range of Output Units

OUTPUT_UNITS

\{from_unit_number, to_unit_number\}

4. Connections Definition

CONNECTIONS

number_of_connections

\{from_unit, to_unit,

weight, delta_weight,

connection_type)

...

\section{Bias Definition}

THRESHOLDS

number_of_units_to_be_defined

\{unit_number,

bias, delta_bias, [required]

integer

[required]

integer

[required]

[integer, integer\}

[required]

integer

(integer, integer

real,real,

binary]

...

[optional]

integer

(integer,

real, real, 
bias_type]

6. Hidden layers Definition

HIDDEN_LAYERS

number_of_hidden_layers

from_unit_number, to_unit_number

7. Driver Definition

DRIVER

number_of_entries_below

\{layer_number, calculation_type,

number_of_connections_in_layer)

B. Simulation Identification and Definition

1. Simulation Name

SIMULATION_NAME

20_char_simulation_name

2. Simulation Type

SIMULATION_TYPE

simulation_type_number

3. Simulation Input Type

INPUT_TYPE

simulation_input_type_number

4. Input/output patterns for training

INPUT_PATTERNS

total_number_of_blocks

\{block_number,

number_of_patterns_in_block\}

[input_pattern\}

...

[teaching_pattern]

5. Simulations to Run

SIMULATIONS

number_of_simulations_to_run

6. Simulation Separator

RUNSIM

C. Report Generation

1. Monitoring Units

PROBE_UNITS

\{flag, number_of_units_specified_below\}

\{unit_number, number_of_sweeps_between_reports\} [optional]

binary\}

...

[required]

integer

[integer, integer]

...

[required]

integer

(integer, integer, integer\}

[optional]

character

[required]

integer

[required]

integer

[required]

integer

\{integer,

integer\}

(integers or reals )

\{integers or reals\}

[required]

integer

[optional]

\{binary, integer\}

[integer, integer\} 


\section{APPENDIX (Continued)}

2. Recording the performance of units over a number of sweeps

\section{RECORD_UNITS}

\{flag,number_of_units_specified_below\}

\{starting_unit_number,ending_unit_number, number_of_sweeps_between_reports

3. Monitoring Connections

\section{PROBE_WEIGHTS}

\{flag, number_of_connections_specified_below [from_unit, to_unit, number_of_sweeps_between_reports $\cdots$

\section{Sweep Monitoring}

PROBE_SWEEPS

[flag, number_of_sweeps_between_reports ]

5. Report weights to be used by PDP program

\section{PDP_REPORT}

flag

6. Generalization Patterns

GENERALIZATION

total_number_of_blocks

(block_number, number_of_patterns_in_block\}

\{generalization_input_pattern\}

‥

[expected_output_pattern]

...

7. Generalization (testing) Report

GENERALIZATION_REPORT

type_of_report

8. Produce Generalization Reports On Demand

REPORTING

number_of_sweeps_between_reports

9. Produce Averages for generalizations performances

$$
\begin{aligned}
& \text { AVERAGES } \\
& \text { \{number_of_clusters_of_patterns_k, } \\
& \text { n_in_cluster_1, n_in_cluster_2, } \\
& \text {..., n_in_cluster_k\} }
\end{aligned}
$$

[optional]

\{binary,integer\}

finteger,integer, integer\}

...

[optional]

(binary, integer)

(integer, integer,

integer\}

‥

[optional]

(binary, integer)

[optional]

binary

[optional]

integer

(integer, integer)

\{integers or reals\}

-..

\{integers or reals\}

[optional]

integer

[optional]

integer

[optional]

[integer,

integer, integer,

..., integer \} 
D. Options

1. Fast/slow weights

FAST_WEIGHTS

\{flag, fast_eta, fast_alpha, real,fast_decay\}

2. Noise

NOISE

\{distribution_type, mean, limit\}

3. Weight retention

KEEP_WEIGHTS

flag

4. Damaging units

MODIFY_UNITS

number_of_units_to_damage

unit_number

...

E. Random Number Control

1. Distribution Type

RANDOM

distribution_type

2. Mean

MEAN

value_of_mean

3. Limit

LIMIT

value_of_limit

4. Seed

SEED

seed_value

F. Learning Parameters

1. Learning rate

ETA

value_of_eta

2 Momentum

ALPHA

value_of_alpha

G. Stopping/Learning Criteria

1. Error Type

ERROR_CRIT

error_type

2. Error Value

ERROR

error_value [optional]

(binary, real,

real\}

[optional]

\{binary, real, real\}

[optional]

binary

[optional]

integer

integer

...

[required]

binary

[required]

real

[required]

real

[optional]

real

[required]

real

[required]

real

[required]

integer

[required]

real 
APPENDIX (Continued)

3. Maximum Number Of Sweeps Allowed

MAXNUM

[required]

maximum_number_of_sweeps_allowed

integer

Note-The status of the keyword is shown in brackets: [required or optional]. If the keyword is required then it must be provided in order for NNS to run correctly. The items that must appear together on a single line (separated by commas) are delimited by braces. Information on keyword status and data types are provided in the rightmost column. The NNS program contains other keywords that are used for development purposes only; such words do not appear in this document. Because this code is still under development, some code segments are redundant. 